\title{
Direct variational determination of the two-electron reduced density matrix for doubly occupied-configuration-interaction wave functions: The influence of three-index $\mathbf{N}$-representability conditions
}

\author{
Diego R. Alcoba, ${ }^{1,2}$ Alicia Torre, ${ }^{3}$ Luis Lain, ${ }^{3}$ Gustavo E. Massaccesi, ${ }^{4}$ Ofelia B. Oña, ${ }^{5}$ \\ Eduardo M. Honoré, ${ }^{1}$ Ward Poelmans, ${ }^{6}$ Dimitri Van Neck, ${ }^{6}$ Patrick Bultinck, ${ }^{7, a)}$ \\ and Stijn De Baerdemacker ${ }^{6}$ \\ ${ }^{1}$ Departamento de Física, Facultad de Ciencias Exactas y Naturales, Universidad de Buenos Aires, \\ Ciudad Universitaria, 1428 Buenos Aires, Argentina \\ ${ }^{2}$ Instituto de Física de Buenos Aires, Consejo Nacional de Investigaciones Científicas y Técnicas, \\ Ciudad Universitaria, 1428 Buenos Aires, Argentina \\ ${ }^{3}$ Departamento de Química Física, Facultad de Ciencia y Tecnología, Universidad del País Vasco. Apdo. 644, \\ E-48080 Bilbao, Spain \\ ${ }^{4}$ Departamento de Ciencias Exactas, Ciclo Básico Común, Universidad de Buenos Aires, Ciudad Universitaria, \\ 1428 Buenos Aires, Argentina \\ ${ }^{5}$ Instituto de Investigaciones Fisicoquímicas Teóricas y Aplicadas, Universidad Nacional de La Plata, \\ CCT La Plata, Consejo Nacional de Investigaciones Científicas y Técnicas. Diag. 113 y 64 (S/N), Sucursal 4, \\ CC 16, 1900 La Plata, Argentina \\ ${ }^{6}$ Center for Molecular Modeling, Ghent University, Technologiepark 903, B-9052 Zwijnaarde, Belgium \\ ${ }^{7}$ Department of Inorganic and Physical Chemistry, Ghent University, Krijgslaan 281 (S3), 9000 Gent, Belgium
}

(Received 10 October 2017; accepted 19 December 2017; published online 9 January 2018)

This work proposes the variational determination of two-electron reduced density matrices corresponding to the ground state of $N$-electron systems within the doubly occupied-configurationinteraction methodology. The $P, Q$, and $G$ two-index $N$-representability conditions have been extended to the $T 1$ and $T 2\left(T 2^{\prime}\right)$ three-index ones and the resulting optimization problem has been addressed using a standard semidefinite program. We report results obtained from the doubly occupiedconfiguration-interaction method, from the two-index constraint variational procedure and from the two- and three-index constraint variational treatment. The discussion of these results along with a study of the computational cost demanded shows the usefulness of our proposal. Published by AIP Publishing. https://doi.org/10.1063/1.5008811

\section{INTRODUCTION}

Although the full configuration interaction (FCI) method provides the exact solutions of the Schrödinger equation corresponding to an $N$-electron system for a chosen subspace, its practical application is limited to small size systems with small basis sets due to its high computational cost. Many approximate procedures have been proposed attempting to reduce that computational effort. Most of these methods express the $N$-electron wave function by means of truncated $N$-electron determinant expansions in which those determinants are selected according to determined criteria. One of these procedures is the doubly occupied-configurationinteraction (DOCI) method, ${ }^{1-6}$ where all $N$-electron determinants involved in the wave function expansion are composed of doubly occupied orbitals, i.e., the seniority number ${ }^{3,7-10}$ of all these determinants is zero. The DOCI method has proven to be a powerful tool to describe systems with strong correlation ${ }^{11,12}$ and many other methods may be derived from it formulating some approximations. ${ }^{13}$ However, although the computational expense of the DOCI method is considerably

a)Electronic mail: Patrick.Bultinck@UGent.be less than that required in the $\mathrm{FCI}$ treatment, the description of medium or large size systems cannot be tackled either by that procedure, as it still scales exponentially albeit in terms of the number of pairs.

As is well known, the two-electron reduced density matrix (2-RDM) constitutes an alternative tool to wave functions to compute the energy. ${ }^{14-17}$ Moreover, the energy of the ground state of an $N$-electron system can be optimized variationally (v2RDM). Within this technique, the 2-RDM matrix elements are optimized subject to constraints so that the resulting 2RDM is $N$-representable (it arises from an $N$-electron wave function). ${ }^{18-21}$ The necessary and sufficient conditions for a 2-RDM to be $N$-representable are known, ${ }^{22-25}$ but in practice, only a limited set of such constraints is used, commonly only the so-called $P, Q$, and $G$ two-index $N$-representability conditions. Recently, we have reported ${ }^{26,27}$ results of the v2RDM method under these $P, Q$, and $G$ conditions approximating the 2-RDM arising from zero seniority number wave functions. We have performed that task formulating the optimization problem as a semidefinite program (SDP) ${ }^{28-31}$ specifically adapted towards $\mathrm{v} 2 \mathrm{RDM}^{21,32,33}$ in which one maximizes a linear function on the intersection of a linear affine space and the convex cone of block-diagonal positive semidefinite matrices. The aim of this work is to extend our previous 
studies $^{26,27}$ and the recent work by Head-Marsden and Mazziotti ${ }^{34}$ by including the $T 1$ and $T 2\left(T 2^{\prime}\right)$ three-index conditions ${ }^{35-37}$ in the v2RDM-DOCI scheme, in order to know the importance of these additional $N$-representability conditions and to study the increase of the computational cost due to the use of the three-index constraints. We report numerical determinations for the ground state potential energy curves obtained from our procedure in selected molecular systems. These results have been compared with those arising from full-DOCI calculations, showing that the use of the threeindex conditions provides a significant improvement upon those obtained using only two-index conditions, albeit still at an affordable computational cost. Moreover, since the DOCI results are not invariant under a unitary orbital transformation, we have performed studies on the basis set dependence of our results.

This work has been organized as follows. In Sec. II, we describe the equations which formulate the two- and three-index $N$-representability conditions, as well as the procedure we have used in this work to deal with the SDP codes within the v2RDM-DOCI approximation. Section III reports the computational details and the results obtained for selected systems with several orbital basis sets, which allows one to discuss the influence of the three-index conditions and the basis set dependence of these results. The computational scaling of our algorithms is also studied in this section. Finally, in Sec. IV, we summarize the main conclusions of this work.

\section{THEORETICAL ASPECTS}

We will formulate the nonrelativistic Hamiltonian of a pairwise-interacting $\mathrm{N}$-electron system in the formalism of second quantization as ${ }^{38}$

$$
\hat{H}=\sum_{i j} h_{j}^{i} a_{i}^{\dagger} a_{j}+\frac{1}{2} \sum_{i j k l} R_{j l}^{i k} a_{i}^{\dagger} a_{k}^{\dagger} a_{l} a_{j},
$$

in which $a_{i}^{\dagger}$ and $a_{j}$ are the standard creation and annihilation fermion operators, respectively, for a given orthonormal $2 K$ spin-orbital basis set $\{i, j, k, l, \ldots\}$ ( $K$ spatial orbitals), $h_{j}^{i}$ are the one-electron integrals (the sum of electron kinetic energy and electron-nucleus potential energy), and $R_{j l}^{i k}=[i j \mid k l]$ stands for the two-electron repulsion ones (in the [11/22] convention).

According to Eq. (1), the electronic energy corresponding to an $N$-electron wave function $\Psi$ of the system is

$$
\begin{aligned}
E(\Psi) & =\sum_{i j} h_{j}^{i}\left\langle\Psi\left|a_{i}^{\dagger} a_{j}\right| \Psi\right\rangle+\sum_{i j k l} R_{j l}^{i k}\left\langle\Psi\left|\frac{a_{i}^{\dagger} a_{k}^{\dagger} a_{l} a_{j}}{2}\right| \Psi\right\rangle \\
& =\operatorname{tr}\left(h^{1} D\right)+\operatorname{tr}\left(R^{2} D\right),
\end{aligned}
$$

in which $\left\langle\Psi\left|a_{i}^{\dagger} a_{j}\right| \Psi\right\rangle$ and $\left\langle\Psi\left|\frac{a_{i}^{\dagger} a_{k}^{\dagger} a_{l} a_{j}}{2}\right| \Psi\right\rangle$ are the 1-RDM and 2RDM elements, respectively, that will be denoted as ${ }^{1} D_{j}^{i}$ and ${ }^{2} D_{j l}^{i k}$.

The numerical determination of the ${ }^{1} D_{j}^{i}$ and ${ }^{2} D_{j l}^{i k}$ elements, for $N$-electron ground $(g)$ states, can be implemented by means of the variational method minimizing the energy $E(\Psi)$ in Eq. (2),

$$
E_{g}=\min _{\left\{{ }^{2} D,{ }^{1} D\right\}}\left(\sum_{i j} h_{j}^{i 1} D_{i}^{j}+\sum_{i j k l} R_{j l}^{i k}{ }^{2} D_{i k}^{j l}\right) .
$$

In this formulation, we have kept the 1-RDM (although this matrix is known if the 2-RDM is known) since, according to Zhao et al. ${ }^{39,40}$ it provides numerically more stable SDP problems.

Although this procedure is the most intuitive method for evaluating the energy $E_{g}$, its results are very often meaningless since such a procedure does not guarantee that the obtained 1-RDM and 2-RDM arise from an $N$-electron wave function. In order to solve this problem, great efforts have been dedicated to search for conditions that the 1-RDM, 2-RDM, and related matrices must satisfy to provide reliable results; this challenge is known as the $N$-representability problem. ${ }^{17-22,35-37,41,42}$ Well-known $N$-representability conditions, which are constraints imposed to the variational method, require that the following matrix elements constitute positive semidefinite matrices

$$
\begin{gathered}
{ }^{1} D_{j}^{i}=\left\langle\Psi\left|a_{i}^{\dagger} a_{j}\right| \Psi\right\rangle, \\
{ }^{1} Q_{j}^{i}=\left\langle\Psi\left|a_{j} a_{i}^{\dagger}\right| \Psi\right\rangle, \\
{ }^{2} P_{j l}^{i k}=\left\langle\Psi\left|a_{i}^{\dagger} a_{k}^{\dagger} a_{l} a_{j}\right| \Psi\right\rangle, \\
{ }^{2} Q_{j l}^{i k}=\left\langle\Psi\left|a_{j} a_{l} a_{k}^{\dagger} a_{i}^{\dagger}\right| \Psi\right\rangle, \\
{ }^{2} G_{j l}^{i k}=\left\langle\Psi\left|a_{i}^{\dagger} a_{k} a_{l}^{\dagger} a_{j}\right| \Psi\right\rangle, \\
(T 1)_{j l n}^{i k m}=\left\langle\Psi\left|a_{i}^{\dagger} a_{k}^{\dagger} a_{m}^{\dagger} a_{n} a_{l} a_{j}+a_{n} a_{l} a_{j} a_{i}^{\dagger} a_{k}^{\dagger} a_{m}^{\dagger}\right| \Psi\right\rangle, \\
(T 2)_{j l n}^{i k m}=\left\langle\Psi\left|a_{i}^{\dagger} a_{k}^{\dagger} a_{m} a_{n}^{\dagger} a_{l} a_{j}+a_{n}^{\dagger} a_{l} a_{j} a_{i}^{\dagger} a_{k}^{\dagger} a_{m}\right| \Psi\right\rangle, \\
\left(T 2^{\prime}\right)=\left(\begin{array}{cc}
T 2 & X \\
X^{\dagger} & 1 \\
D
\end{array}\right) .
\end{gathered}
$$

Equations (4) and (5) describe the matrix elements of the one-electron and one-electron hole reduced density matrices, respectively. The matrix elements ${ }^{1} Q_{j}^{i}$ can be calculated by means of those of the 1-RDM ones, using the anticommutation rules of fermion operators. The positive semidefiniteness of both matrices ${ }^{1} D$ and ${ }^{1} Q$ and the $\operatorname{tr}\left({ }^{1} D\right)$ value constitute the Coleman necessary and sufficient ensemble $N$-representability conditions for the 1-RDM. ${ }^{18,19}$ The matrices described in Eqs. (6)-(8) require the use of two indices (two creation and two annihilation operators). They constitute the above mentioned $P, Q$, and $G$ conditions, respectively, requiring that the corresponding matrix turns out to be positive semidefinite. Likewise, the $T 1$ and $T 2\left(T 2^{\prime}\right)$ conditions mean that the three-index matrices shown in Eqs. (9) and (10) or (9) and (11) must also be positive semidefinite. The matrix elements of all these matrices can be expressed in terms of only the 1-RDM and 2-RDM; the anticommutation rules of the fermion operators lead to 3-RDM elements that cancel in the $T 1$ and $T 2\left(T 2^{\prime}\right)$ expressions. The $T 2^{\prime}$ condition, in which $X_{j l n}^{p}=2{ }^{2} D_{j l}^{p n}$, is stronger than the $T 2$ one and can replace it entirely. ${ }^{43,44}$ Apart from these conditions, the matrices ${ }^{1} D,{ }^{1} Q,{ }^{2} P,{ }^{2} Q,{ }^{2} G, T 1$, and $T 2\left(T 2^{\prime}\right)$ must be Hermitian and satisfy the antisymmetric conditions $^{36}$ 


$$
{ }^{2} D_{j l}^{i k}=-{ }^{2} D_{j l}^{k i}=-{ }^{2} D_{l j}^{i k} .
$$

Similarly, the matrices ${ }^{2} Q$ and $T 1$ must be antisymmetric with respect to all pair and triple indices, respectively, and the matrix $T 2\left(T 2^{\prime}\right)$ must be antisymmetric with respect to the first 2 indices of each trio.

In this work, the one- and two-electron reduced density matrices have been normalized according to

$$
\begin{gathered}
\operatorname{tr}\left({ }^{1} D\right)=N, \\
\operatorname{tr}\left({ }^{2} D\right)=\left(\begin{array}{l}
N \\
2
\end{array}\right),
\end{gathered}
$$

and, consequently, the contraction relation between both matrices is

$$
{ }^{1} D_{i}^{j}=\frac{2}{N-1} \sum_{k}{ }^{2} D_{j k}^{i k}
$$

As mentioned in the Introduction, we will only refer to DOCI wave functions. These wave functions are eigenstates of the Hamiltonian projected over the Hilbert space restricted to Slater determinants where all spatial orbitals are doubly occupied, or, equivalently, of the effective Hamiltonian

$$
\begin{aligned}
& \hat{H}^{e f f}=\sum_{\sigma} \sum_{i^{\sigma}} h_{i^{\sigma}}^{i^{\sigma}} a_{i^{\sigma}}^{\dagger} a_{i \sigma}+\frac{1}{2} \sum_{\sigma} \sum_{i^{\sigma} \neq j^{\sigma}} R_{i^{\sigma} j^{\sigma}}^{i^{\sigma} j^{\sigma}} a_{i^{\sigma}}^{\dagger} a_{j^{\sigma}}^{\dagger} a_{j \sigma} a_{i} \\
& +\frac{1}{2} \sum_{\sigma} \sum_{i^{\sigma}, j^{\bar{\sigma}}} R_{i^{\sigma} j^{\bar{\sigma}}}^{i^{\sigma} \bar{\sigma}} a_{i^{\sigma}}^{\dagger} a_{j^{\bar{\sigma}}}^{\dagger} a_{j \bar{\sigma}} a_{i^{\sigma}} \\
& +\frac{1}{2} \sum_{\sigma} \sum_{i^{\sigma} \neq j^{\sigma}} R_{j^{\sigma} i^{\sigma}}^{i^{\sigma} j^{\sigma}} a_{i^{\sigma}}^{\dagger} a_{j^{\sigma}}^{\dagger} a_{i^{\sigma}} a_{j^{\sigma}} \\
& +\frac{1}{2} \sum_{\sigma} \sum_{i^{\sigma} \neq j^{\sigma}} R_{j^{\sigma} j^{\bar{\sigma}}}^{i^{\sigma} i^{\bar{\sigma}}} a_{i^{\sigma}}^{\dagger} a_{i^{\bar{\sigma}}}^{\dagger} a_{j \bar{\sigma} \bar{\sigma}} a_{j \sigma},
\end{aligned}
$$

in which we denote by $\sigma$ the spin coordinate $(\alpha$ or $\beta$ ) of the corresponding orbital and $\bar{\sigma}$ means its spin conjugate. This effective Hamiltonian $\hat{H}^{\text {eff }}$ contains much less terms than $\hat{H}$ in Eq. (1) although both of them provide identical information for DOCI wave functions. According to Eq. (16), only the matrix elements ${ }^{1} D_{i^{\sigma}}^{i^{\sigma}},{ }^{2} D_{i^{\sigma} j^{\sigma}}^{i^{\sigma} j^{\sigma}},{ }^{2} D_{i^{\sigma} j^{\sigma}}^{i^{\sigma} j^{\bar{\sigma}}},{ }^{2} D_{j^{\sigma} i^{\sigma}}^{i^{\sigma_{j}}}$, and ${ }^{2} D_{j \sigma}^{i \sigma^{\sigma} i^{\bar{\sigma}}}$ need to be determined for energy calculations; the remainder 1-RDM and 2-RDM ones are zero within the DOCI framework.

The DOCI wave functions are linear combinations of $N$-electron determinants which are eigenfunctions of the $N$ electron $\hat{S}^{2}$ operator ${ }^{45}$ corresponding to a $S=0$ spin quantum number, and consequently these wave functions are singlet states with an expectation value equal to zero for the seniority number operator $\hat{\Omega}, 3,9$

$$
\langle\hat{\Omega}\rangle_{\mathrm{DOCI}}=\sum_{\sigma} \sum_{i^{\sigma}}{ }^{1} D_{i^{\sigma}}^{i^{\sigma}}-2 \sum_{\sigma} \sum_{i^{\sigma}}{ }^{2} D_{i^{\sigma} i^{\bar{\sigma}}}^{i^{\sigma} i^{\bar{\sigma}}}=0 .
$$

This condition or equivalently

$$
\sum_{\sigma} \sum_{i^{\sigma}}{ }^{2} D_{i^{\sigma} i^{\sigma^{\sigma}}}^{i^{\sigma} \bar{\sigma}^{\bar{\sigma}}}=\frac{N}{2}
$$

along with the traces of the spin blocks of the 1-RDM and 2RDM, suffices to enforce the singlet and seniority-zero (DOCI) character of the corresponding wave functions. Alternatively to using the effective Hamiltonian (16), the "full" Hamiltonian can be used along with DOCI specific $N$-representability con-

\begin{tabular}{|c|c|c|c|c|c|c|c|c|c|}
\hline & & \multicolumn{4}{|c|}{$\operatorname{Max} \mathrm{AE}\left(E_{h}\right)$} & \multicolumn{4}{|c|}{$\operatorname{NPE}\left(E_{h}\right)$} \\
\hline & & $P Q G$ & $P Q G T 1$ & $P Q G T 1 T 2$ & $P Q G T 1 T 2^{\prime}$ & $P Q G$ & $P Q G T 1$ & $P Q G T 1 T 2$ & $P Q G T 1 T 2^{\prime}$ \\
\hline \multirow[t]{4}{*}{$\mathrm{N}_{2}$} & $\mathrm{CMO}$ & 0.0017 & 0.0007 & $<0.0001$ & $<0.0001$ & 0.0017 & 0.0007 & $<0.0001$ & $<0.0001$ \\
\hline & NO & 0.0011 & 0.0004 & $<0.0001$ & $<0.0001$ & 0.0010 & 0.0004 & $<0.0001$ & $<0.0001$ \\
\hline & $\mathrm{M}_{\min }$ & 0.0011 & 0.0005 & $<0.0001$ & $<0.0001$ & 0.0011 & 0.0004 & $<0.0001$ & $<0.0001$ \\
\hline & OPTE & 0.0012 & 0.0005 & $<0.0001$ & $<0.0001$ & 0.0011 & 0.0004 & $<0.0001$ & $<0.0001$ \\
\hline \multirow[t]{4}{*}{$\mathrm{CO}$} & $\mathrm{CMO}$ & 0.0344 & 0.0197 & 0.0034 & 0.0034 & 0.0343 & 0.0196 & 0.0034 & 0.0034 \\
\hline & NO & 0.1022 & 0.0815 & 0.0334 & 0.0334 & 0.1020 & 0.0814 & 0.0333 & 0.0333 \\
\hline & $\mathrm{M}_{\min }$ & 0.0360 & 0.0178 & 0.0042 & 0.0042 & 0.0358 & 0.0177 & 0.0042 & 0.0042 \\
\hline & OPTE & 0.0111 & 0.0031 & 0.0011 & 0.0011 & 0.0108 & 0.0030 & 0.0011 & 0.0011 \\
\hline \multirow[t]{4}{*}{$\mathrm{CN}^{-}$} & $\mathrm{CMO}$ & 0.0940 & 0.0506 & 0.0114 & 0.0114 & 0.0939 & 0.0505 & 0.0114 & 0.0114 \\
\hline & NO & 0.0145 & 0.0127 & 0.0006 & 0.0006 & 0.0143 & 0.0126 & 0.0006 & 0.0006 \\
\hline & $\mathrm{M}_{\min }$ & 0.0097 & 0.0083 & 0.0003 & 0.0003 & 0.0096 & 0.0083 & 0.0003 & 0.0003 \\
\hline & OPTE & 0.0038 & 0.0028 & 0.0002 & 0.0002 & 0.0036 & 0.0028 & 0.0002 & 0.0002 \\
\hline \multirow[t]{4}{*}{$\mathrm{NO}^{+}$} & $\mathrm{CMO}$ & 0.1050 & 0.0523 & 0.0119 & 0.0119 & 0.1049 & 0.0523 & 0.0119 & 0.0119 \\
\hline & NO & 0.0207 & 0.0181 & 0.0009 & 0.0009 & 0.0205 & 0.0180 & 0.0008 & 0.0008 \\
\hline & $\mathrm{M}_{\min }$ & 0.0132 & 0.0115 & 0.0005 & 0.0005 & 0.0130 & 0.0115 & 0.0005 & 0.0005 \\
\hline & OPTE & 0.0042 & 0.0035 & 0.0003 & 0.0003 & 0.0040 & 0.0035 & 0.0003 & 0.0002 \\
\hline
\end{tabular}
straints. These have been formulated previously by Weinhold and Wilson ${ }^{1,2}$ and extended further more recently ${ }^{26,27,34}$ (see also the Appendix).

TABLE I. Maximum Absolute (MaxAE) and Nonparallelity (NPE) errors of the ground state potential energy curves of molecules and ions calculated by the v2RDM-DOCI method imposing the $P Q G, P Q G T 1, P Q G T 1 T 2$, and $P Q G T 1 T 2^{\prime}$ conditions with respect to the DOCI results using the canonical molecular orbitals (CMOs), natural orbitals (NOs), minimizing the FCI seniority number orbitals $\left(\mathrm{M}_{\min }\right)$, and optimizing the energy orbitals (OPTEs). Curves are computed in the interval $[1.4,4.0]$ bohrs. Results were obtained using the STO-3G atomic basis set. 
We have formulated the variational RDM method as a standard dual SDP problem modifying the procedure described by Zhao et al. ${ }^{36}$ to use the SDP codes so that our algorithms satisfy Eq. (18). The determination of the 1-RDM and 2-RDM elements corresponding to DOCI wave functions has been performed only by using the one- and two-electron integrals defining the effective Hamiltonian described by Eq. (16), i.e., the diagonal elements of the one-electron integrals and the one- and two-index repulsion integrals, respectively. The SDP codes employed ${ }^{46}$ solve semidefinite problems at several precision levels by means of the Mehrotratype predictor-corrector infeasible primal-dual interior-point method, ${ }^{47}$ providing electronic energies and 1-RDM and 2-RDM.

\section{COMPUTATIONAL DETAILS, RESULTS, AND DISCUSSION}

We have chosen the set of isoelectronic systems $\mathrm{N}_{2}, \mathrm{CO}$, $\mathrm{CN}^{-}$, and $\mathrm{NO}^{+}$to test the behavior of our proposals, determining their ground-state potential energy curves. All the reported results have been obtained from the STO-3G atomic basis sets, in order to limit the computational cost. As is well known, the resulting DOCI energy is not invariant with respect to a unitary transformation of the orthonormal basis set used and the same applies to v2RDM-DOCI. ${ }^{26,27,34}$ To study the basis set dependence of our results, we have performed calculations using the following orthonormal basis sets: (i) the canonical molecular orbitals (CMOs), (ii) the natural orbitals (NOs), (iii) the orbitals which minimize the seniority number of the FCI expansions $\left(\mathrm{M}_{\mathrm{min}}\right),{ }^{9}$ and (iv) the orbitals which minimize the DOCI energy (OPTE). ${ }^{12}$ The PSI3.4 package ${ }^{48}$ has been used to obtain the one- and twoelectron integrals expressed in the CMO basis sets. We have used our own codes to construct and to diagonalize either the standard Hamiltonian matrix, to obtain the $\mathrm{NO}$ and $\mathrm{M}_{\min }$ basis sets, or its projection in the DOCI space to obtain the OPTE ones. The full-DOCI treatment (energies and RDMs) has been

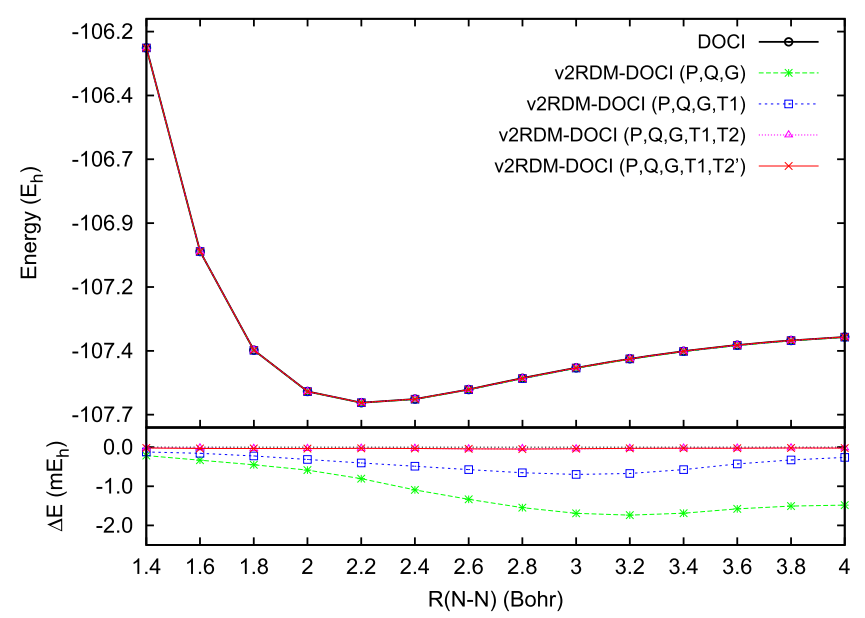

FIG. 1. Ground state potential energy curve of the $\mathrm{N}_{2}$ molecule calculated by the DOCI and v2RDM-DOCI procedures imposing the $P Q G, P Q G T 1$, $P Q G T 1 T 2$, and $P Q G T 1 T 2^{\prime}$ conditions. Energy errors, $\triangle \mathrm{E}$, relative to reference DOCI values. Results were obtained using the canonical molecular orbitals arising from the STO-3G atomic basis set.

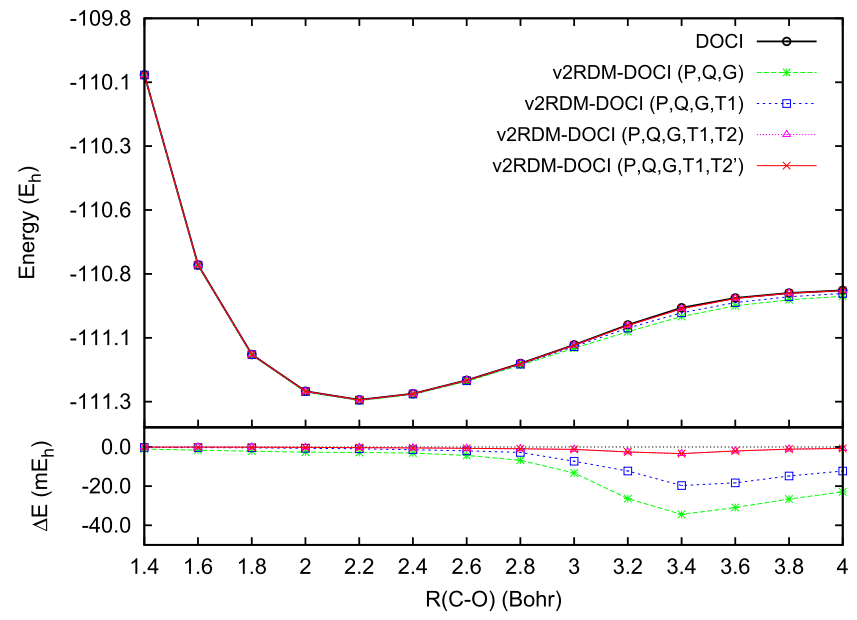

FIG. 2. Ground state potential energy curve of the $\mathrm{CO}$ molecule calculated by the DOCI and v2RDM-DOCI procedures imposing the $P Q G, P Q G T 1$, $P Q G T 1 T 2$, and $P Q G T 1 T 2^{\prime}$ conditions. Energy errors, $\triangle \mathrm{E}$, relative to reference DOCI values. Results were obtained using the canonical molecular orbitals arising from the STO-3G atomic basis set.

implemented using modified versions of the algorithms reported in Refs. 49 and 50. We have elaborated codes that allow to efficiently solve the SDP algorithms identifying and exploiting the sparse matrix data structure of the $P, Q, G, T 1$, and $T 2\left(T 2^{\prime}\right)$ matrices induced by the structure of the seniorityzero wave functions. The SDPA 7.3.8 code $^{46}$ was used to provide the corresponding v2RDM-DOCI energy values and 1-RDM and 2-RDM elements.

In Table I, we report the maximum absolute errors (MaxAE) found between the energies obtained from the use of different $\mathrm{N}$-representability conditions in the variational method, and the full-DOCI energy, as a function of the internuclear distance, using several orthonormal orbital basis sets.

As can be seen, in all cases, the imposition of the $T 1$ and $T 2\left(T 2^{\prime}\right)$ conditions on top of the $P, Q$, and $G$ ones significantly reduces these energy differences. Table I also shows

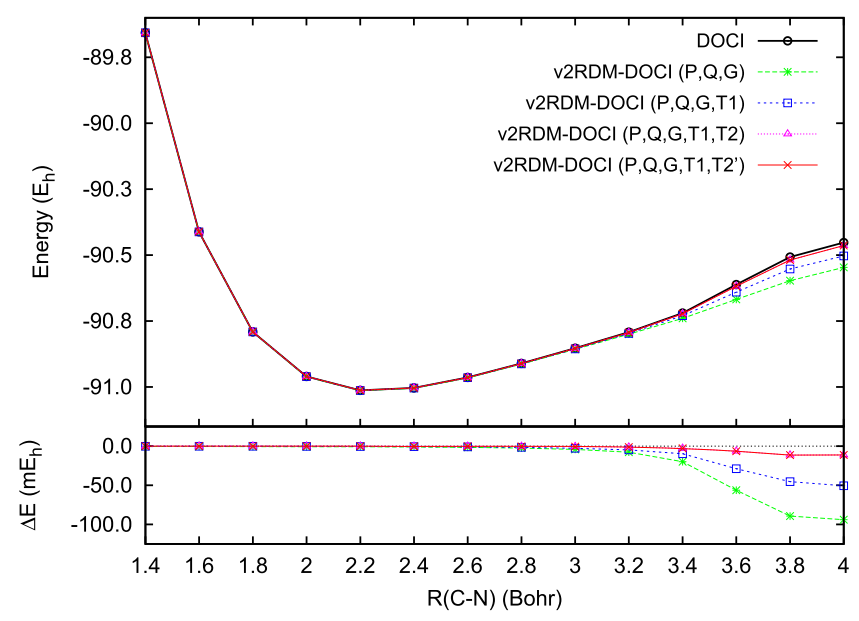

FIG. 3. Ground state potential energy curve of the $\mathrm{CN}^{-}$molecule calculated by the DOCI and v2RDM-DOCI procedures imposing the PQG, PQGT1, $P Q G T 1 T 2$, and $P Q G T 1 T 2^{\prime}$ conditions. Energy errors, $\triangle \mathrm{E}$, relative to reference DOCI values. Results were obtained using the canonical molecular orbitals arising from the STO-3G atomic basis set. 


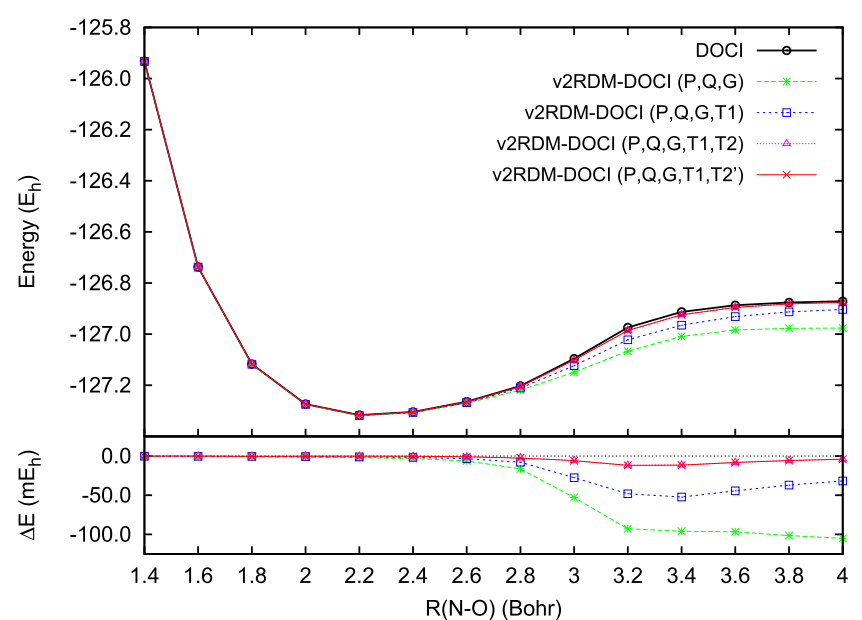

FIG. 4. Ground state potential energy curve of the $\mathrm{NO}^{+}$molecule calculated by the DOCI and v2RDM-DOCI procedures imposing the $P Q G, P Q G T 1$, $P Q G T 1 T 2$, and $P Q G T 1 T 2^{\prime}$ conditions. Energy errors, $\triangle \mathrm{E}$, relative to reference DOCI values. Results were obtained using the canonical molecular orbitals arising from the STO-3G atomic basis set.

the nonparallelity errors (NPEs) in that interval, that is, the differences between the maximum and minimum deviation from the full-DOCI energy values. Both MaxAE and NPE quantities predict similar behavior, having very similar numerical values. Figures 1-4 show the potential energy curves obtained with the CMO basis sets for the studied systems, as well as the energy errors, $\Delta \mathrm{E}$, relative to the corresponding reference DOCI values.

These curves point out that the $P Q G T 1 T 2^{\prime}$ variational method yields the closest values to the full-DOCI one, in agreement with the results exhibited in Table I. It is worth noting that the $T 1$ and $T 2\left(T 2^{\prime}\right)$ conditions strongly reduce the $P Q G$ non-parallelity error near bond dissociation. Moreover, the comparative analysis of the counterpart values arising from the different molecular orbital basis sets, reported in Table I, shows that the OPTE one leads to the best results, the $\mathrm{M}_{\min }$ basis set

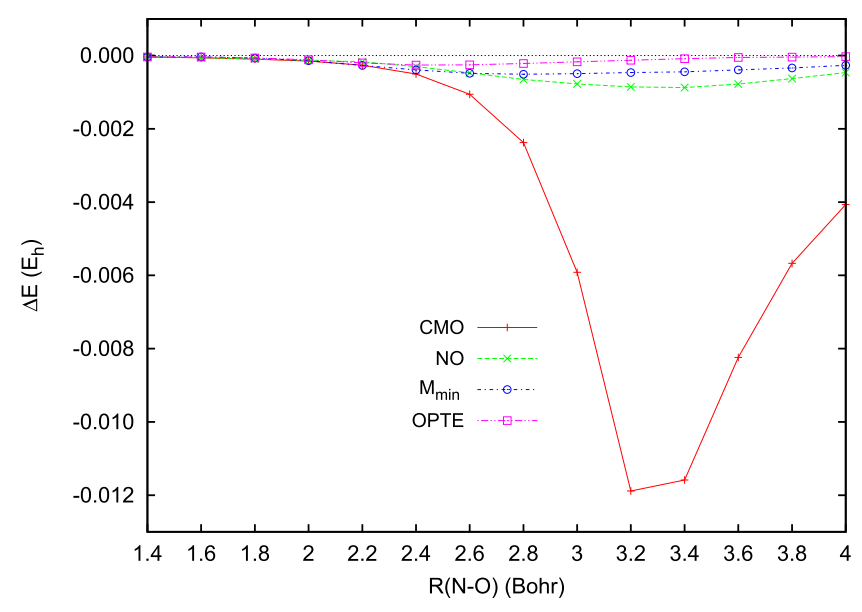

FIG. 5. Errors for the $\mathrm{NO}^{+}$ground state energies calculated by the v2RDMDOCI procedure imposing the $P Q G T 1 T 2^{\prime}$ conditions, with respect to the corresponding DOCI values. Results were obtained using the canonical molecular orbitals (CMOs), natural orbitals (NOs), orbitals which minimize the seniority number of the FCI wave functions $\left(\mathrm{M}_{\min }\right)$, and orbitals which minimize the DOCI energy (OPTE) arising from the STO-3G atomic basis set.

presents better behavior than the natural orbitals, while the CMO basis set provides a poor approximation. Similar conclusions can be drawn from Fig. 5, which shows the errors for the $\mathrm{NO}^{+}$ground state energies calculated for all those orthonormal basis sets by the $P Q G T 1 T 2^{\prime}$ variational method with respect to the reference DOCI energy values.

We have gathered in Table II the values of the energy differences $\mathrm{E}_{\mathrm{v} 2 \mathrm{RDM}-\mathrm{DOCI}}-\mathrm{E}_{\mathrm{DOCI}}$ corresponding to the $\mathrm{NO}^{+}$ system obtained for each of the above mentioned orthonormal basis sets by imposing the $P, Q, G, T 1$, and $T 2\left(T 2^{\prime}\right) \mathrm{N}$ representability conditions. These values have been obtained at both an internuclear distance near the equilibrium (2.2 bohrs) and at a stretched geometry (4.0 bohrs). These results again show a clear improvement according to the basis set sequence $\mathrm{CMO}<\mathrm{NO}<\mathrm{M}_{\min }<\mathrm{OPTE}$ as well as with the imposed conditions series $P Q G<P Q G T 1<P Q G T 1 T 2$; no

TABLE II. Energy errors in the ground state of the $\mathrm{NO}^{+}$molecule at two bond lengths calculated in the canonical molecular orbitals (CMOs), natural orbitals (NOs), orbitals which minimize the seniority number of the FCI wave functions $\left(\mathrm{M}_{\min }\right)$ and orbitals which minimize the DOCI energy (OPTE). The errors calculated by the DOCI variational RDM method (v2RDM-DOCI) are computed imposing the $P Q G, P Q G T 1, P Q G T 1 T 2$, and $P Q G T 1 T 2^{\prime}$ conditions. Results were obtained using the STO-3G atomic basis set.

\begin{tabular}{|c|c|c|c|c|c|}
\hline & \multirow[t]{2}{*}{$\mathrm{E}_{\mathrm{DOCI}}\left(E_{h}\right)$} & \multicolumn{4}{|c|}{$\mathrm{E}_{\mathrm{v} 2 \mathrm{RDM}-\mathrm{DOCI}}-\mathrm{E}_{\mathrm{DOCI}}\left(E_{h}\right)$} \\
\hline & & $P Q G$ & $P Q G T 1$ & $P Q G T 1 T 2$ & $P Q G T 1 T 2^{\prime}$ \\
\hline & \multicolumn{5}{|c|}{$\mathrm{R}_{\mathrm{eq}}=2.2 \mathrm{bohrs}$} \\
\hline $\mathrm{CMO}$ & -127.316934 & $-2.12 \times 10^{-3}$ & $-1.09 \times 10^{-3}$ & $-2.57 \times 10^{-4}$ & $-2.57 \times 10^{-4}$ \\
\hline NO & -127.320704 & $-1.99 \times 10^{-3}$ & $-7.30 \times 10^{-4}$ & $-1.71 \times 10^{-4}$ & $-1.71 \times 10^{-4}$ \\
\hline $\mathrm{M}_{\min }$ & -127.324924 & $-3.91 \times 10^{-3}$ & $-1.02 \times 10^{-3}$ & $-2.70 \times 10^{-4}$ & $-2.69 \times 10^{-4}$ \\
\hline \multirow[t]{2}{*}{ OPTE } & -127.326027 & $-2.80 \times 10^{-3}$ & $-7.96 \times 10^{-4}$ & $-2.13 \times 10^{-4}$ & $-2.01 \times 10^{-4}$ \\
\hline & \multicolumn{5}{|c|}{$\mathrm{R}_{\mathrm{st}}=4.0$ bohrs } \\
\hline $\mathrm{CMO}$ & -126.871538 & $-1.05 \times 10^{-1}$ & $-3.18 \times 10^{-2}$ & $-4.07 \times 10^{-3}$ & $-4.07 \times 10^{-3}$ \\
\hline NO & -127.119269 & $-1.79 \times 10^{-2}$ & $-1.26 \times 10^{-2}$ & $-4.63 \times 10^{-4}$ & $-4.62 \times 10^{-4}$ \\
\hline $\mathrm{M}_{\min }$ & -127.121756 & $-1.24 \times 10^{-2}$ & $-8.90 \times 10^{-3}$ & $-2.59 \times 10^{-4}$ & $-2.59 \times 10^{-4}$ \\
\hline OPTE & -127.128085 & $-1.29 \times 10^{-3}$ & $-9.96 \times 10^{-4}$ & $-2.68 \times 10^{-5}$ & $-2.68 \times 10^{-5}$ \\
\hline
\end{tabular}


TABLE III. 1-RDM errors in the $\mathrm{NO}^{+}$molecule at two bond lengths, calculated for the v2RDM-DOCI method in the canonical molecular orbitals (CMOs), natural orbitals (NOs), orbitals which minimize the seniority number of the FCI wave functions $\left(\mathrm{M}_{\min }\right)$ and orbitals which minimize the DOCI energy (OPTE). The errors are computed imposing the $P Q G, P Q G T 1$, $P Q G T 1 T 2$, and $P Q G T 1 T 2^{\prime}$ conditions. Results were obtained using the STO-3G atomic basis set.

\begin{tabular}{|c|c|c|c|c|}
\hline & \multicolumn{4}{|c|}{$\sqrt{\frac{\sum_{i=1}^{K}\left[\sum_{\sigma}{ }^{1} D_{i}^{i^{i} \sigma}(\mathrm{v} 2 \mathrm{RDM}-\mathrm{DOCI})-\sum_{\sigma}{ }^{1} D_{i}^{i \sigma}(\mathrm{DOCl})\right]^{2}}{K}}$} \\
\hline & $P Q G$ & $P Q G T 1$ & $P Q G T 1 T 2$ & $P Q G T 1 T 2^{\prime}$ \\
\hline & \multicolumn{4}{|c|}{$\mathrm{R}_{\mathrm{eq}}=2.2 \mathrm{bohrs}$} \\
\hline $\mathrm{CMO}$ & 0.002 & 0.001 & $<0.001$ & $<0.001$ \\
\hline NO & 0.002 & 0.001 & $<0.001$ & $<0.001$ \\
\hline $\mathrm{M}_{\min }$ & 0.003 & 0.001 & $<0.001$ & $<0.001$ \\
\hline \multirow[t]{2}{*}{ OPTE } & 0.002 & 0.001 & $<0.001$ & $<0.001$ \\
\hline & \multicolumn{4}{|c|}{$\mathrm{R}_{\mathrm{st}}=4.0 \mathrm{bohrs}$} \\
\hline $\mathrm{CMO}$ & 0.239 & 0.191 & 0.017 & 0.017 \\
\hline NO & 0.031 & 0.022 & $<0.001$ & $<0.001$ \\
\hline $\mathrm{M}_{\min }$ & 0.021 & 0.016 & $<0.001$ & $<0.001$ \\
\hline OPTE & 0.002 & 0.002 & $<0.001$ & $<0.001$ \\
\hline
\end{tabular}

significant differences have been found between the $P Q G T 1 T 2$ and $P Q G T 1 T 2^{\prime}$ procedures. To complete this study, we report in Table III values of the quantity $\sqrt{\frac{\sum_{i=1}^{K}\left[\sum_{\sigma^{1}} D_{i \sigma}^{i^{\sigma}}(\mathrm{v} 2 \mathrm{RDM}-\mathrm{DOCI})-\sum_{\sigma}{ }^{1} D_{i^{\sigma}}^{i^{\sigma}}(\mathrm{DOCI})\right]^{2}}{K}}$, i.e., the rootmean-square deviation of ${ }^{1} D_{\text {v2RDM-DOCI }}$ with respect to ${ }^{1} D_{\text {DOCI }}$, in terms of the spin-free matrices, for the $\mathrm{NO}^{+}$system at two internuclear distances. The deviation from zero of this quantity measures the mean error of the results. As can be observed, these values confirm, in terms of differences of 1-RDM elements, the conclusions arising from the numerical values found in terms of energy differences, showing similar behavior.

In order to test whether or not the atomic basis set does not have a significant effect on the results, we report in Tables IV and V the energy differences between v2RDM-DOCI and DOCI for the imposed conditions series $P Q G, P Q G T 1$, and $P Q G T 1 T 2$ and for different basis sets. The results for the energies and their comparison with those reported in Table II
TABLE V. 1-RDM errors in the $\mathrm{NO}^{+}$molecule at two bond lengths, calculated for the v2RDM-DOCI method in the canonical molecular orbitals (CMOs). The errors are computed imposing the $P Q G, P Q G T 1, P Q G T 1 T 2$, and $P Q G T 1 T 2^{\prime}$ conditions. Results were obtained using the $3-21 \mathrm{G}$ and DZ atomic basis sets.

\begin{tabular}{|c|c|c|c|c|}
\hline & \multicolumn{4}{|c|}{ 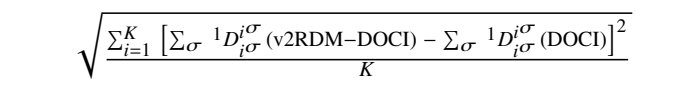 } \\
\hline & $P Q G$ & $P Q G T 1$ & $P Q G T 1 T 2$ & $P Q G T 1 T 2^{\prime}$ \\
\hline & \multicolumn{4}{|c|}{$\mathrm{R}_{\mathrm{eq}}=2.2$ bohrs } \\
\hline $3-21 G$ & 0.001 & 0.001 & $<0.001$ & $<0.001$ \\
\hline \multirow[t]{2}{*}{ DZ } & 0.001 & 0.001 & $<0.001$ & $<0.001$ \\
\hline & \multicolumn{4}{|c|}{$\mathrm{R}_{\mathrm{st}}=4.0 \mathrm{bohrs}$} \\
\hline $3-21 G$ & 0.149 & 0.056 & 0.005 & 0.005 \\
\hline DZ & 0.113 & 0.030 & 0.003 & 0.003 \\
\hline
\end{tabular}

show that there is no drastic influence when using the CMO orthonormal basis and equilibrium geometry. At stretched geometry, the larger basis sets give somewhat smaller energy differences. In Table V, the differences in the 1-RDM and their comparison with those reported in Table III also show that the influence is limited although there the data suggest that there is a small improvement for the larger basis sets.

To evaluate and to compare the computational cost of all procedures used in this work, we have studied the series of linear $\mathrm{H}_{2 \mathrm{n}}$ chains composed of $\mathrm{n}=5-25$ equidistant hydrogen atoms separated by a distance of 2.0 bohrs. The STO-3G basis set has been used so that there is one orbital on each hydrogen atom. In Fig. 6, we plot the computing time against the number of basis functions (or number of hydrogen atoms) both in logarithmic scales. The results show linear behavior in all cases with a similar slope $\beta$, which gives the computational scaling $K^{\beta}$. This scaling is due to the sparse structure of the $T 1$ and $T 2\left(T 2^{\prime}\right)$ matrices within the DOCI framework (see the Appendix), which possess $O\left(K^{3}\right)$ blocks of $O(1 \times 1)$ dimension and $O(K)$ blocks of $O(K \times K)$ dimension, ${ }^{27}$ in contrast to the sparse structure of the $P, Q$, and $G$ matrices which present $O\left(K^{2}\right)$ blocks of $O(1 \times 1)$ dimension and $O(1)$ blocks of $O(K \times K)$ dimension. ${ }^{26}$ Nevertheless, the theoretical

TABLE IV. Energy errors in the ground state of the $\mathrm{NO}^{+}$molecule at two bond lengths calculated in the canonical molecular orbitals (CMOs). The errors calculated by the DOCI variational RDM method (v2RDM-DOCI) are computed imposing the $P Q G, P Q G T 1, P Q G T 1 T 2$, and $P Q G T 1 T 2^{\prime}$ conditions. Results were obtained using the $3-21 \mathrm{G}$ and $\mathrm{DZ}$ atomic basis sets.

\begin{tabular}{|c|c|c|c|c|c|}
\hline & \multirow[t]{2}{*}{$\mathrm{E}_{\mathrm{DOCI}}\left(E_{h}\right)$} & \multicolumn{4}{|c|}{$\mathrm{E}_{\mathrm{V} 2 \mathrm{RDM}-\mathrm{DOCI}}-\mathrm{E}_{\mathrm{DOCI}}\left(E_{h}\right)$} \\
\hline & & $P Q G$ & $P Q G T 1$ & $P Q G T 1 T 2$ & $P Q G T 1 T 2^{\prime}$ \\
\hline & \multicolumn{5}{|c|}{$\mathrm{R}_{\mathrm{eq}}=2.2 \mathrm{bohrs}$} \\
\hline $3-21 G$ & -128.210837 & $-3.55 \times 10^{-3}$ & $-2.00 \times 10^{-3}$ & $-3.48 \times 10^{-4}$ & $-3.46 \times 10^{-4}$ \\
\hline \multirow[t]{2}{*}{$\mathrm{DZ}$} & -128.914169 & $-2.46 \times 10^{-3}$ & $-1.23 \times 10^{-3}$ & $-3.31 \times 10^{-4}$ & $-3.30 \times 10^{-4}$ \\
\hline & \multicolumn{5}{|c|}{$\mathrm{R}_{\mathrm{st}}=4.0 \mathrm{bohrs}$} \\
\hline $3-21 G$ & -127.818809 & $-4.65 \times 10^{-2}$ & $-1.46 \times 10^{-2}$ & $-2.92 \times 10^{-3}$ & $-2.92 \times 10^{-3}$ \\
\hline $\mathrm{DZ}$ & -128.518539 & $-3.11 \times 10^{-2}$ & $-9.97 \times 10^{-3}$ & $-2.02 \times 10^{-3}$ & $-2.02 \times 10^{-3}$ \\
\hline
\end{tabular}




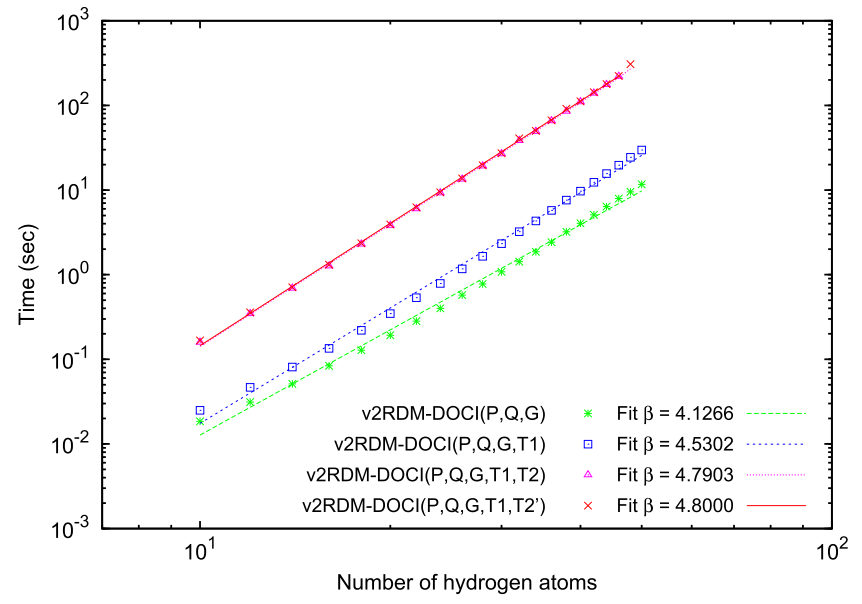

FIG. 6. Scaling of the DOCI variational RDM method (v2RDM-DOCI) imposing the $P Q G, P Q G T 1, P Q G T 1 T 2$, and $P Q G T 1 T 2^{\prime}$ conditions on growing linear chains of equidistant hydrogen atoms $(\mathrm{R}(\mathrm{H}-\mathrm{H})=2.0 \mathrm{bohrs})$ in the STO-3G basis on a $\log -\log$ plot. Data fitted with linear function $(\mathrm{y}=\alpha+\beta \mathrm{x})$.

scaling of the v2RDM-DOCI is $O\left(K^{3}\right)$ for the two-index conditions due to the diagonalisation of $K \times K$ blocks and $O\left(K^{4}\right)$ for the three-index ones, which is three and five orders of magnitude lower than in the regular v2RDM, method respectively. Consequently, from a computational point of view, the addition of the $T 1$ and $T 2\left(T 2^{\prime}\right) N$-representability conditions to the $P, Q$, and $G$ ones in the DOCI framework entails an affordable increase of computational effort, providing a significant improvement of the results.

\section{CONCLUDING REMARKS}

In this work, we have studied the influence of the threeindex $T 1$ and $T 2\left(T 2^{\prime}\right)$ conditions that, added to the two-index $P, Q$, and $G$ ones, constitute necessary constraints to ensure the $N$-representability in the variational determination of the twoelectron reduced density matrix within the DOCI methodology (wave functions possessing a zero seniority number). The results obtained prove an important improvement approaching those provided by the reference method (full-DOCI method). Although this achievement requires an increase of computational cost, the quality of the numerical determinations found promotes the usefulness of the three-index variational constraints within the DOCI framework.

\section{ACKNOWLEDGMENTS}

The authors acknowledge financial support from Universidad de Buenos Aires (Grant No. 20020150100157BA), the Consejo Nacional de Investigaciones Científicas y Técnicas (Grant Nos. PIP 11220130100377CO, PIP 11220130100311CO, and 2013-1401PCB), the Agencia Nacional de Promoción Científica y Tecnológica, Argentina (Grant No. PICT-201-0381), the Universidad del Pais Vasco (Project No. EHU16/10), and the Research Foundation Flanders (FWO Vlaanderen). The authors would also like to thank Dr. B. Verstichel for useful discussions on the DOCI three index conditions.

\section{APPENDIX: CONSTRAINTS ARISING FROM THE THREE-INDEX $N$-REPRESENTABILITY CONDITIONS FOR DOCI WAVE FUNCTIONS}

The operators in identical position of the creation and annihilation strings, which define the reduced density matrices, possess the same spin coordinate. According to this property, one can express the $(T 1)_{j l n}^{i k m}$ matrix, in Eq. (9), as a direct sum of spin blocks $(T 1)_{j^{\alpha} l^{\alpha} n^{\alpha}}^{i^{\alpha} k^{\alpha} m^{\alpha}},(T 1)_{j^{\alpha} l^{\alpha} n^{\beta}}^{i^{\alpha} k^{\alpha} m^{\beta}},(T 1)_{j^{\alpha} l^{\beta} n^{\beta}}^{i^{\alpha} k^{\beta} m^{\beta}}$, and $(T 1)_{j \beta}^{i^{\beta} k^{\beta} m^{\beta}}$.

The $(T 1)_{j^{\alpha} l^{\alpha} n^{\alpha}}^{i^{\alpha} k^{\alpha} m^{\alpha}}$ block has no repetition of indices in the creation (or annihilation) string, otherwise the Pauli principle would be violated. For the DOCI case, the sets $\{i, k, m\}$ and $\{j$, $l, n\}$ must be constituted by identical indices so that all nonzero matrix elements of that block are equal (in absolute value) to the corresponding diagonal elements, which are nonnegative. According to Eq. (9), one straightforwardly finds

$$
\begin{aligned}
(T 1)_{i^{\alpha} k^{\alpha} m^{\alpha}}^{i^{\alpha} k^{\alpha} m^{\alpha}}= & 1-{ }^{1} D_{i^{\alpha}}^{i^{\alpha}}-{ }^{1} D_{k^{\alpha}}^{k^{\alpha}}-{ }^{1} D_{m^{\alpha}}^{m^{\alpha}}+2{ }^{2} D_{i^{\alpha} k^{\alpha}}^{i^{\alpha} k^{\alpha}} \\
& +2{ }^{2} D_{k^{\alpha} m^{\alpha}}^{k^{\alpha} m^{\alpha}}+2^{2} D_{i^{\alpha} m^{\alpha}}^{i^{\alpha} m^{\alpha}} \geq 0
\end{aligned}
$$

This constraint ${ }^{2}$ leads to $K(K-1)(K-2) / 6$ diagonal conditions. A similar set of constraints can be obtained for the $(T 1)_{i^{\beta} k^{\beta} m^{\beta}}^{i^{\beta} m^{\beta} m^{\beta}}$ block.

The matrix elements of the $(T 1)_{j^{\alpha} l^{\alpha} n^{\beta}}^{i^{\alpha} k^{\alpha} m^{\beta}}$ block for DOCI wave functions and different $i, k$, and $m$ indices can also be reduced to the diagonal ones. Consequently, one finds new $K(K-1)(K-2) / 2$ diagonal conditions,

$$
\begin{aligned}
(T 1)_{i^{\alpha} k^{\alpha} m^{\beta}}^{i^{\alpha} k^{\alpha} m^{\beta}}= & 1-{ }^{1} D_{i^{\alpha}}^{i^{\alpha}}-{ }^{1} D_{k^{\alpha}}^{k^{\alpha}}-{ }^{1} D_{m^{\beta}}^{m^{\beta}}+2{ }^{2} D_{i^{\alpha} k^{\alpha}}^{i^{\alpha} k^{\alpha}} \\
& +2{ }^{2} D_{k^{\alpha} m^{\beta}}^{k^{\alpha} m^{\beta}}+2{ }^{2} D_{i^{\alpha} m^{\beta}}^{i^{\alpha} m^{\beta}} \geq 0
\end{aligned}
$$

It is possible, however, that the index $m$ may be equal to the $i$ or $k$ index. Using the anticommutation rules for the fermion operators, it is sufficient to analyze the case $k=m$. In this case, the DOCI conditions require that the $j$ or $l$ indices are equal to $i$, but it is again sufficient to consider the case $i=j$. Moreover, in this case, we have that $l=n$. Hence, we must analyze the blocks $(T 1)_{i^{\alpha}}^{i^{\alpha} k^{\alpha} l^{\beta}} \forall i$, and therefore we find $K$ blocks of size $(K-1) \times(K-1)$ since $k, l \neq i$,

$$
(T 1)_{i^{\alpha} l^{\alpha} l^{\beta}}^{i^{\alpha} k^{\alpha} k^{\beta}}=\delta_{l}^{k}\left(1-2{ }^{1} D_{k^{\alpha}}^{k^{\alpha}}-{ }^{1} D_{i^{\alpha}}^{i^{\alpha}}+2{ }^{2} D_{k^{\alpha} i^{\alpha}}^{k^{\alpha} i^{\alpha}}\right)+2{ }^{2} D_{k^{\alpha} k^{\beta}}^{l^{\alpha} l^{\beta}},
$$

where each of the terms in this expression defines a $(K-1) \times$ $(K-1)$ positive semidefinite matrix $\forall i$. This is a new constraint which has no counterpart in Ref. 2. A similar set of constraints can be obtained for the $(T 1)_{i^{\alpha} k^{\beta} m^{\beta}}^{i^{\alpha} k^{\beta} m^{\beta}}$ block. The $T 2$ constraints are found as

$$
\begin{gathered}
(T 2)_{j l n}^{i k m}=\left\langle\psi\left|a_{i}^{\dagger} a_{k}^{\dagger} a_{m} a_{n}^{\dagger} a_{l} a_{j}+a_{n}^{\dagger} a_{l} a_{j} a_{i}^{\dagger} a_{k}^{\dagger} a_{m}\right| \psi\right\rangle, \\
(X)_{j \mathrm{ln}}^{p}=2{ }^{2} D_{j l}^{p n}=\left\langle\psi\left|a_{p}^{\dagger} a_{n}^{\dagger} a_{l} a_{j}\right| \psi\right\rangle, \\
{ }^{1} D_{q}^{p}=\left\langle\psi\left|a_{p}^{\dagger} a_{q}\right| \psi\right\rangle .
\end{gathered}
$$


In a block matrix form, the $T 2^{\prime}$ condition can be expressed as

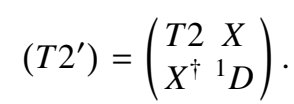

In the $T 2$ case, and due to the antisymmetry in the first and second indices of this matrix and the singlet character of the DOCI wavefunctions, there are two types of blocks to be considered. One of these types of blocks corresponds for instance to the blocks generated by the operators $a_{i^{\alpha}}^{\dagger} a_{k^{\alpha}}^{\dagger} a_{m^{\beta}}, a_{m^{\alpha}}^{\dagger} a_{i^{\alpha}}^{\dagger} a_{k^{\beta}}$, and $a_{k^{\alpha}}^{\dagger} a_{m^{\alpha}}^{\dagger} a_{i \beta}$ for every ordered combination of different $i, k$, and $m$. In this case, it follows that

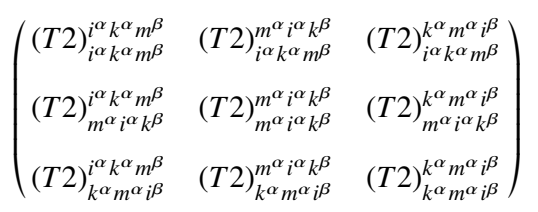

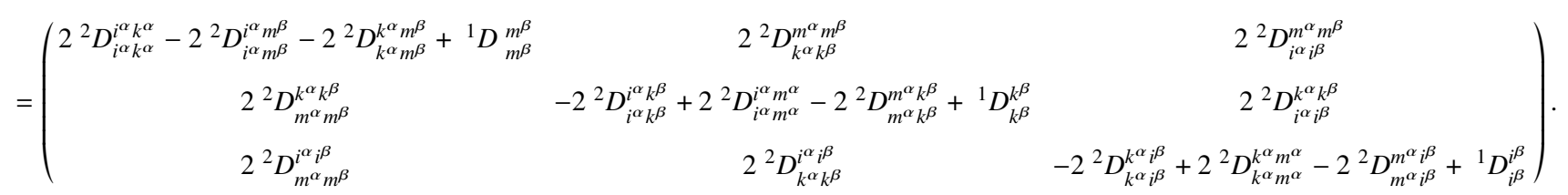

It is possible to carry out similar calculations for the blocks corresponding to other spin combinations. These blocks have similar expressions and the same numerical values, except for the signs that are due to the basis selection and are not relevant to the positivity of these blocks. Hence, we find $2 K(K-1)(K-2) / 3$ blocks of size $3 \times 3$.

The second type of blocks corresponds for instance to the blocks generated by the operators $a_{i^{\alpha}}^{\dagger} a_{k^{\alpha}}^{\dagger} a_{k^{\alpha}}, a_{i^{\alpha}}^{\dagger} a_{k^{\beta}}^{\dagger} a_{k^{\beta}}, a_{k^{\alpha}}^{\dagger} a_{k^{\beta}}^{\dagger} a_{i^{\beta}}$, and $a_{i^{\alpha}}^{\dagger} a_{i \beta}^{\dagger} a_{i \beta}$ for each index $i$. The selection of the operators avoids those that can be skipped due to the antisymmetric properties of $T 2$. Also, in this list, $k$ represents all indices different from $i$, so there are $3(K-1)+1$ combinations. In this case, it follows that

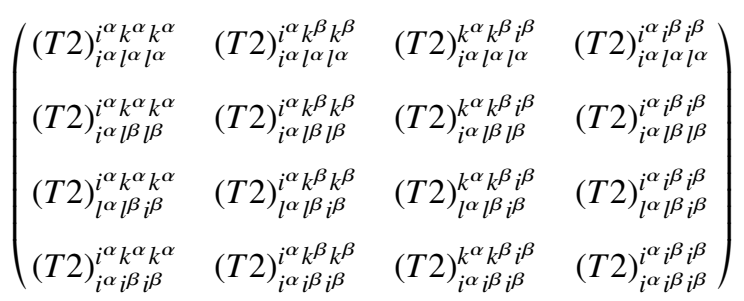

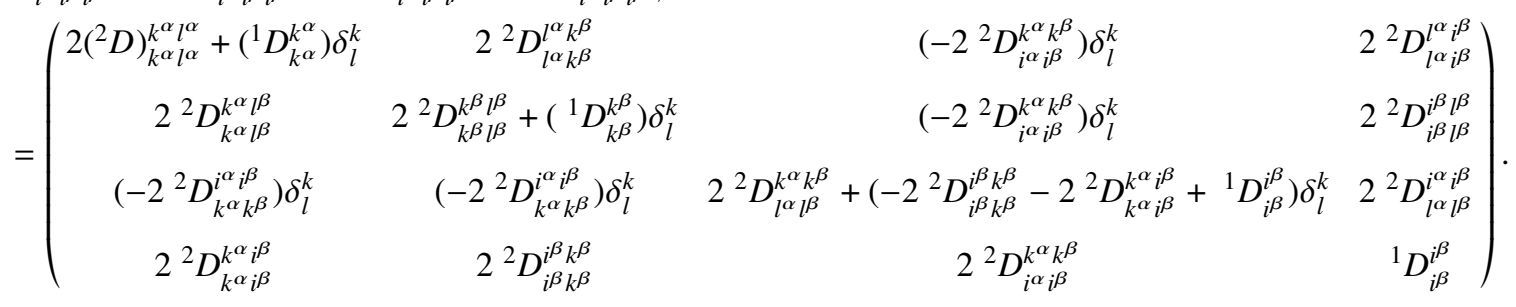

Taking into account those blocks corresponding to other spin combinations, we find $2 K$ blocks of size $(3(K-1)+1)$ $\times(3(K-1)+1)$.

Similar analysis can be carried out for the $T 2^{\prime}$ condition. In this case, one finds $2 K(K-1)(K-2) / 3$ blocks of size $3 \times 3$ and $2 K$ blocks of size $(3(K-1)+2) \times(3(K-1)+2)$. These constraints and those arising from the $T 2\left(T 2^{\prime}\right)$ conditions have been reported by Poelmans. ${ }^{27}$

${ }^{1}$ F. Weinhold and E. B. Wilson, J. Chem. Phys. 46, 2752 (1967).

${ }^{2}$ F. Weinhold and E. B. Wilson, J. Chem. Phys. 47, 2298 (1967).

${ }^{3}$ L. Bytautas, T. M. Henderson, C. A. Jimenez-Hoyos, J. K. Ellis, and G. E. Scuseria, J. Chem. Phys. 135, 044119 (2011).

${ }^{4}$ P. A. Limacher, P. W. Ayers, P. A. Johnson, S. De Baerdemacker, D. Van Neck, and P. Bultinck, J. Chem. Theory Comput. 9, 1394 (2013).

${ }^{5}$ T. Stein, T. M. Henderson, and G. E. Scuseria, J. Chem. Phys. 140, 214113 (2014).
${ }^{6}$ P. A. Limacher, T. D. Kim, P. W. Ayers, P. A. Johnson, S. De Baerdemacker, D. Van Neck, and P. Bultinck, Mol. Phys. 112, 853 (2014).

${ }^{7}$ P. Ring and P. Schuck, The Nuclear Many-Body Problem (Springer-Verlag, Berlin, Heidelberg, 1980).

${ }^{8}$ P. Karafiloglou, J. Chem. Phys. 130, 164103 (2009).

${ }^{9}$ D. R. Alcoba, A. Torre, L. Lain, G. E. Massaccesi, and O. B. Ona, J. Chem. Phys. 139, 084103 (2013).

${ }^{10}$ D. R. Alcoba, A. Torre, L. Lain, G. E. Massaccesi, and O. B. Ona, J. Chem. Phys. 140, 234103 (2014).

${ }^{11}$ D. R. Alcoba, A. Torre, L. Lain, O. B. Ona, P. Capuzzi, M. Van Raemdonck, P. Bultinck, and D. Van Neck, J. Chem. Phys. 141, 244118 (2014).

${ }^{12}$ M. Van Raemdonck, D. R. Alcoba, W. Poelmans, S. De Baerdemacker, A. Torre, L. Lain, G. E. Massaccesi, D. Van Neck, and P. Bultinck, J. Chem. Phys. 143, 104106 (2015).

${ }^{13}$ C. Zhou, Z. Chen, and W. Wu, Comput. Theor. Chem. 1116, 86 (2017).

${ }^{14}$ K. Husimi, Proc. Physico-Mat. Soc. Japan, 3rd Series 22, 264 (1940).

${ }^{15}$ P. O. Lowdin, Phys. Rev. 97, 1474 (1955).

${ }^{16}$ J. E. Mayer, Phys. Rev. 100, 1579 (1955). 
${ }^{17}$ C. A. Coulson, Rev. Mod. Phys. 32, 170 (1960).

${ }^{18}$ A. J. Coleman, Rev. Mod. Phys. 35, 668 (1963).

${ }^{19}$ A. J. Coleman, Int. J. Quantum Chem. 11, 907 (1977).

${ }^{20}$ Reduced-Density-Matrix Mechanics: With Applications to Many-Electron Atoms and Molecules, Advances in Chemical Physics, edited by D. A. Mazziotti (John Wiley \& Sons, Inc., 2007), Vol. 134.

${ }^{21}$ M. Nakata, H. Nakatsuji, M. Ehara, M. Fukuda, K. Nakata, and K. Fujisawa, J. Chem. Phys. 114, 8282 (2001).

${ }^{22}$ C. Garrod and J. K. Percus, J. Math. Phys. 5, 1756 (1964).

${ }^{23}$ H. Kummer, J. Math. Phys. 8, 2063 (1967).

${ }^{24}$ A. J. Coleman, J. Math. Phys. 13, 214 (1972).

${ }^{25}$ D. A. Mazziotti, Phys. Rev. Lett. 108, 263002 (2012).

${ }^{26}$ W. Poelmans, M. Van Raemdonck, B. Verstichel, S. De Baerdemacker, A. Torre, L. Lain, G. E. Massaccesi, D. R. Alcoba, P. Bultinck, and D. Van Neck, J. Chem. Theory Comput. 11, 4064 (2015).

${ }^{27}$ W. Poelmans, "Variational determination of the two-particle density matrix: The case of doubly-occupied space," Ph.D. thesis, Ghent University, 2015.

${ }^{28}$ Y. Nesterov and A. Nemirovskii, Interior Point Polynomial Method in Convex Programming: Theory and Applications (SIAM, Philadelphia, 1993).

${ }^{29}$ L. Vandenberghe and S. Boyd, SIAM Rev. 38, 49 (1996).

${ }^{30}$ S. Wright, Primal-Dual Interior-Point Methods (SIAM, Philadelphia, 1997).

${ }^{31}$ Handbook of Semidefinite Programming: Theory, Algorithms, and Applications, International Series in Operations Research \& Management Science, edited by H. Wolkowicz, R. Saigal, and L. Vandenberghe (Springer Science+Business Media, New York, 2000), Vol. 27.

${ }^{32}$ D. A. Mazziotti, Phys. Rev. A 65, 062511 (2002).

${ }^{33}$ B. Verstichel, H. van Aggelen, D. Van Neck, P. Bultinck, and S. De Baerdemacker, Comput. Phys. Commun. 182, 1235 (2011).

${ }^{34}$ K. Head-Marsden and D. A. Mazziotti, J. Chem. Phys. 147, 084101 (2017).

${ }^{35}$ R. M. Erdahl, Int. J. Quantum Chem. 13, 697 (1978).
${ }^{36}$ Z. J. Zhao, B. J. Braams, M. Fukuda, M. L. Overton, and J. K. Percus, J. Chem. Phys. 120, 2095 (2004).

${ }^{37}$ D. A. Mazziotti, Phys. Rev. A 72, 032510 (2005).

${ }^{38}$ P. Jorgensen and J. Simons, Second Quantization-Based Methods in Quantum Chemistry (Academic Press, New York, 1981).

${ }^{39} \mathrm{Z}$. Zhao, "The reduced density matrix method for electronic structure calculations: Application of semidefinite programming to N-fermion systems," Ph.D. thesis, New York University, 2004.

${ }^{40}$ M. Fukuda, B. J. Braams, M. Nakata, M. L. Overton, J. K. Percus, M. Yamashita, and Z. Zhao, Math.Program. 109, 553 (2007).

${ }^{41}$ R. M. Erdahl and B. Jin, J. Mol. Struct.: THEOCHEM 527, 207 (2000).

${ }^{42}$ D. A. Mazziotti and R. M. Erdahl, Phys. Rev. A 63, 042113 (2001).

${ }^{43}$ B. J. Braams, J. Percus, and Z. Zhao, "The T1 and T2 representability conditions," Reduced-Density-Matrix Mechanics: With Applications To Many-Electron Atoms and Molecules (Wiley-Interscience, 2007), Chap. 5, pp. 93-101.

${ }^{44}$ G. Gidofalvi and D. A. Mazziotti, J. Chem. Phys. 126, 024105 (2007).

${ }^{45}$ A. Torre and L. Lain, J. Mol. Struct.: THEOCHEM 426, 25 (1998); xXIIIrd International Congress of Theoretical Chemists of Latin Expression, Caceres, Spain, Sep 16-20, 1996.

${ }^{46}$ M. Yamashita, K. Fujisawa, M. Fukuda, K. Kobayashi, K. Nakata, and M. Nakata, "Latest developments in the SDPA family for solving large-scale SDPs," Handbook on Semidefinite, Conic and Polynomial Optimization (Springer International Publishing AG, 2012), Vol. 166, pp. 687-713.

${ }^{47}$ M. Nakata, B. J. Braams, K. Fujisawa, M. Fukuda, J. K. Percus, M. Yamashita, and Z. Zhao, J. Chem. Phys. 128, 164113 (2008).

${ }^{48}$ T. D. Crawford, C. D. Sherrill, E. F. Valeev, J. T. Fermann, R. A. King, M. L. Leininger, S. T. Brown, C. L. Janssen, E. T. Seidl, J. P. Kenny, and W. D. Allen, J. Comput. Chem. 28, 1610 (2007).

${ }^{49}$ G. Knizia and G. K.-L. Chan, Phys. Rev. Lett. 109, 186404 (2012).

${ }^{50}$ G. Knizia and G. K.-L. Chan, J. Chem. Theory Comput. 9, 1428 (2013). 